\title{
Enhanced Third Harmonic Generation in Single Germanium Nanodisks Excited at the Anapole Mode
}

Gustavo Grinblat, Yi Li, Michael P. Nielsen, Rupert F. Oulton, and Stefan

\author{
A. Maier
}

The Blackett Laboratory, Department of Physics, Imperial College London, London SW7 AZ, United Kingdom

Corresponding to: g.grinblat@imperial.ac.uk; yi.li@imperial.ac.uk 


\section{1- Fabrication of germanium nanodisks}

Arrays of germanium nanodisks were fabricated on borosilicate glass using electron beam lithography [1-4]. The substrate was first coated with positive-tone PMMA (950K A4) resist, and posteriorly baked at $180{ }^{\circ} \mathrm{C}$ for $5 \mathrm{~min}$. Then the nanostructures were defined by an electron beam exposure, followed by a development procedure. Germanium thermal evaporation at $1.5 \AA / s$, and lift-off processes were the final steps. Figure S1a summarizes the fabrication procedure, while Figure S1b shows the final array for germanium disks with $875 \mathrm{~nm}$ diameter.

(a)
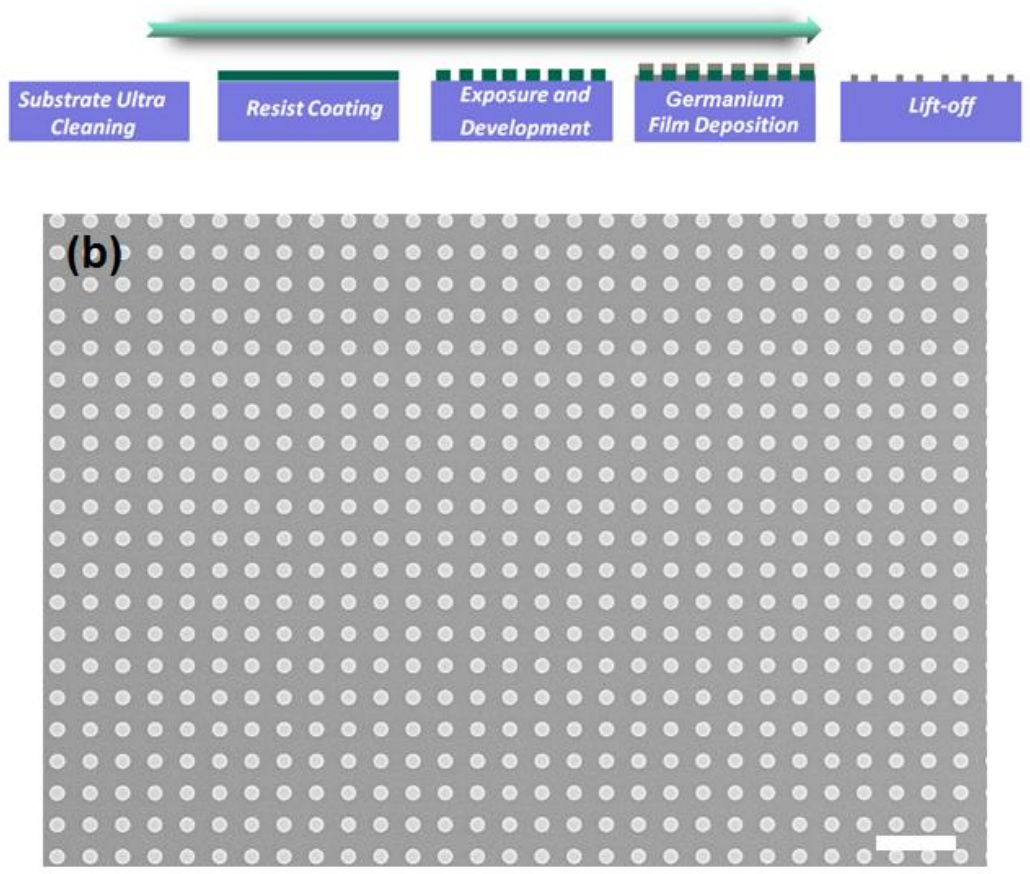

Figure S1. (a) An illustration of fabrication steps for the array of germanium nanodisks. (b) SEM image for the case of germanium disks with $875 \mathrm{~nm}$ diameter. Scale bar, $5 \mu \mathrm{m}$. 


\section{2- Optical measurements details}

Extinction measurements were obtained by Fourier transform infrared spectroscopy (FTIR, Bruker Hyperion 2000) at normal incidence with linear polarization in transmission mode.

THG (third harmonic generation) characterizations were performed through the experimental setup sketched in Figure S2. A pulsed $\mathrm{Yb}$ :KGW PHAROS laser system was used as the pump of a collinear optical parametric amplifier ORPHEUS with a LYRA wavelength extension option (Light Conversion Ltd, pulse duration $180 \mathrm{fs}$, repetition rate $100 \mathrm{kHz})$. The excitation beam $(\lambda=1550 \mathrm{~nm}-1750 \mathrm{~nm})$ was reflected by a shortpass dichroic mirror (Thorlabs DMSP1000) and focused onto the sample with a 100X (NA = 0.9) air objective from Nikon. The third harmonic emission was collected in a backscattering configuration via the same objective, and detected with an avalanche photodiode (MPD PDM Series by Picoquant) for imaging, or by a spectrograph (PI Acton SP2300 by Princeton Instruments) for spectral measurements. The sample was fixed to a XYZ piezo-scanner stage (Nano-Drive, Mad City Labs) to perform the scanning.

The conversion efficiency was determined by directly measuring the collected third harmonic emission power with a calibrated Si photodetector (Newport), and the excitation power with a Ge photodetector (Thorlabs), and then computing the ratio between the both. For $<1 \mathrm{pW}$ third harmonic powers, values were calibrated by using the measured third harmonic spectra.

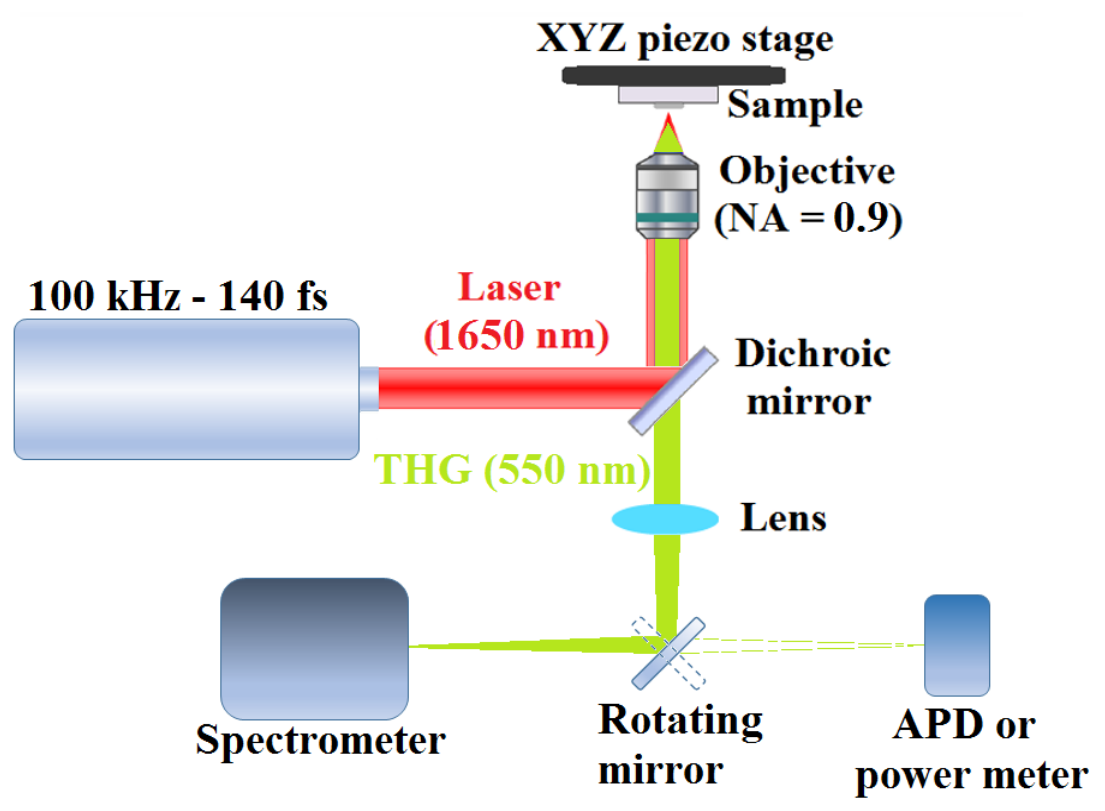

Figure S2. Schematic diagram of the microscope used for the THG studies. 


\section{3- Z-scan characterization}

The third order nonlinear characterization of unstructured germanium and silicon was conducted using a Z-scan setup, as seen in Figure S3a, using the same laser system used in the THG experiments at $\lambda=1650 \mathrm{~nm}$. To avoid heating the samples, the laser repetition rate was reduced to $100 \mathrm{~Hz}$. A small portion of the laser beam was sent to a reference InGaAs photodiode (Newport 818-IG) to remove power fluctuations from the measurements. The remaining light was focused to a focal spot of $37.5 \mu \mathrm{m}$ and then the whole beam was imaged on a 2-D InGaAs CCD array (Xenics Xeva-1.7-320) in the far field. The two-photon absorption coefficient $\beta_{T P A}$ was measured by studying how the total transmission through the sample changed while passing through the focus of the beam, called an open aperture measurement, through the following equation $[5,6]$ :

$$
\Delta T(z)=\frac{-q_{o}}{2 \sqrt{2}} \frac{1}{\left(1+^{z^{2}} / z_{R}^{2}\right)}, q_{o}=\beta_{T P A} I_{o} L_{e f f}
$$

where $\Delta T(z)$ is the change in transmission of the sample, $z_{R}$ is the Rayleigh range, $I_{o}$ is the peak intensity at the focus, and $L_{e f f}$ is the effective sample thickness after absorption is taken into account. By analyzing the transmission in a small region in the center of the beam, known as a closed aperture measurement, it is possible to determine the optical Kerr coefficient $n_{2}$ through [5,6]:

$$
\begin{gathered}
\Delta T_{p-v}=0.406(1-S)^{0.27}\left|\Delta \Phi_{\mathrm{o}}\right| \\
\left|\Delta \Phi_{o}\right|=\frac{2 \pi}{\lambda} n_{2} I_{o} L_{e f f}
\end{gathered}
$$

where $\Delta T_{p-v}$ is the difference between the peak and the valley of the scan, $S$ is the portion of the power of the beam analyzed, and $\left|\Delta \Phi_{o}\right|$ is the nonlinear phase shift. When both two-photon absorption and nonlinear refraction occur simultaneously, the open aperture scan must be divided by the closed aperture scan to remove the effects of two-photon absorption from the nonlinear refraction. When the two-photon absorption is significantly strong, a more sensitive method must be used, such as eclipse Z-scan method [7], where only the edge of the beam is analyzed. In this case:

$$
\Delta T_{p-v}=0.68(1-S)^{-0.44}\left|\Delta \Phi_{\mathrm{o}}\right|
$$

where in this method $S$ is the amount of power of the beam rejected from the analysis and $\left|\Delta \Phi_{o}\right|$ is the same as in the closed aperture case. For eclipse Z-scan to be accurate, the care must be made such that $S \geq 0.98$ and $\left|\Delta \Phi_{o}\right| \leq 0.2$. It should be noted that for eclipse Z-scan, the location of the peak and the valley are reversed compared to a closed 
aperture scan for a given sign of $n_{2}$. Once both $n_{2}$ and $\beta_{T P A}$ are known, the third order susceptibility $\chi^{(3)}$ for third harmonic generation can be calculated with [8,9]:

$$
\begin{gathered}
\frac{\omega}{c} n_{2}(\omega)+\frac{i}{2} \beta_{T P A}(\omega)=\frac{3 \omega}{4 \epsilon_{o} c^{2} n_{o}^{2}} \chi_{1111}^{(3)}(-\omega ; \omega,-\omega, \omega) \\
\chi_{1111}^{(3)}(3 \omega ; \omega, \omega, \omega)=\frac{1}{3} \chi_{1111}^{(3)}(-\omega ; \omega,-\omega, \omega) \\
\chi^{(3)}[S I]=\left(\frac{4 \pi}{9} \times 10^{-8}\right) \chi^{(3)}[e s u]
\end{gathered}
$$

The Z-scan results of a double-side polished $300 \mu \mathrm{m}$-thick silicon substrate can be seen in Figure S3b. For this measurement, the intensity at the focus was $3.56 \times 10^{13} \mathrm{~W} / \mathrm{m}^{2}$ and $S=0.07$. This gives $n_{2, \text { Si@ } 1650 \mathrm{~nm}}=2.69 \times 10^{-18} \mathrm{~m}^{2} / W$ and $\beta_{T P A, S i @ 1650 \mathrm{~nm}}=$ $1.56 \mathrm{~cm} / G W$, in agreement with the literature $[10,11]$. From this, the real part of the third order nonlinear susceptibility is obtained, $\chi_{3 \omega, \text { Si@1650nm }}^{(3)}=2.75 \times 10^{-12}$ esu.

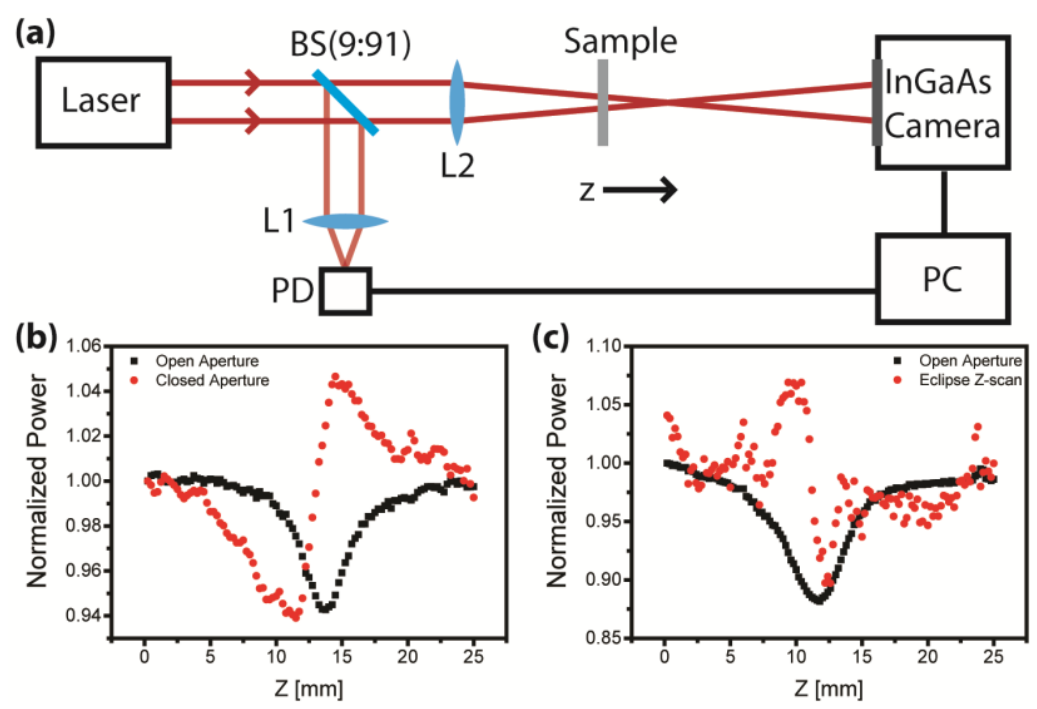

Figure S3. (a) Schematic of the Z-scan measurements. The beam passes through a 9:91 R:T beamsplitter (BS), wherein the reflected portion is focused through a lens L1 of $\mathrm{f}=50 \mathrm{~mm}$ on a photodiode (PD) as a reference power detector. The remaining laser light is focused through a lens L2 of $\mathrm{f}=250 \mathrm{~mm}$ and imaged on a 2-D InGaAs CCD array. The sample is then passed through the focus of the light and the distortions of the beam are imaged on the CCD array and analyzed on the PC. (b) Z-scan results of a double-side polished $300 \mu \mathrm{m}$-thick silicon substrate illustrating open and closed aperture results. (b) Z-scan results of a $1.6 \mu \mathrm{m}$-thick germanium film on a $130 \mu \mathrm{m}$ thick silica substrate illustrating open aperture and eclipse Z-scan results.

Due to the significant two-photon absorption in germanium, eclipse Z-scan was required to measure its Kerr index. The results of a $1.6 \mu \mathrm{m}$-thick germanium film on a silica substrate can be seen in Figure S3c. For this measurement, the intensity at the focus was $7.71 \times 10^{13} \mathrm{~W} / \mathrm{m}^{2}$ and $S=0.98$. The obtained values are $n_{2, G e @ 1650 \mathrm{~nm}}=2.67 \times$ $10^{-17} \mathrm{~m}^{2} / W$ and $\beta_{T P A, G e @ 1650 \mathrm{~nm}}=289 \mathrm{~cm} / G W$. This results in a real part of the third order nonlinear susceptibility $\chi_{3 \omega, G e @ 1650 \mathrm{~nm}}^{(3)}=4.05 \times 10^{-11} \mathrm{esu}$. 


\section{4- Magnetic field distribution at the anapole mode}

Figure S4 shows $\mathrm{XY}$ and $\mathrm{ZY}$ cross sections of the simulated magnetic field distribution $|\mathrm{H}| /\left|\mathrm{H}_{0}\right|$ for a germanium disk of $875 \mathrm{~nm}$ diameter and $100 \mathrm{~nm}$ height excited at the anapole mode. We note that the distribution matches that reported for the silicon case [12].
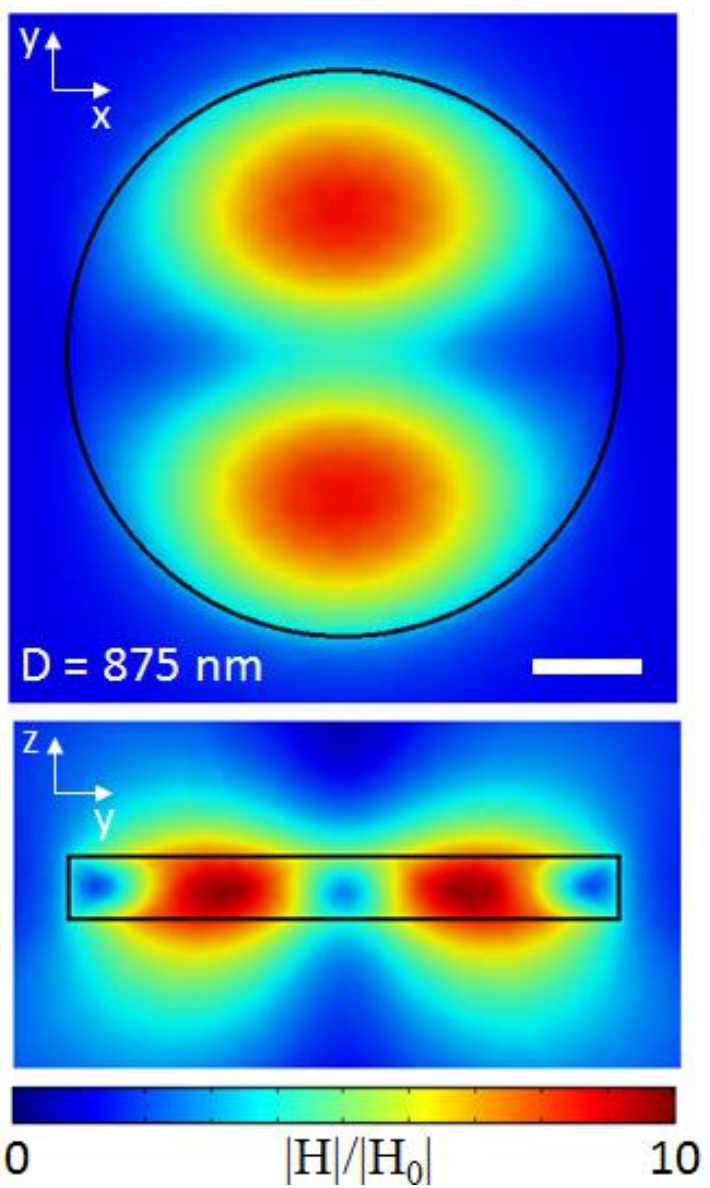

Figure S4. Calculated magnetic field distribution $\left(|\mathrm{H}| /\left|\mathrm{H}_{0}\right|\right)$ at the anapole mode for $\mathrm{D}=875 \mathrm{~nm}$, for incident light linearly polarized in the x-direction. Scale bar, $200 \mathrm{~nm}$. 


\section{5- Simulations for germanium disks of different thicknesses}

Figure S5a exhibits the simulated scattering cross section for a disk of $500 \mathrm{~nm}$ diameter (D) and $100 \mathrm{~nm}$ height $(\mathrm{t})$. The nanostructure supports the anapole mode as evidenced by the corresponding inner electric energy (Figure S5b) and electric and magnetic fields distributions. When $t$ is doubled (i.e. $t=200 \mathrm{~nm}$ ), the scattering cross section changes dramatically (Figure S5c), and the electric energy presents the maximum value at the magnetic dipole resonance, as revealed by the fields distributions (Figure S5d).
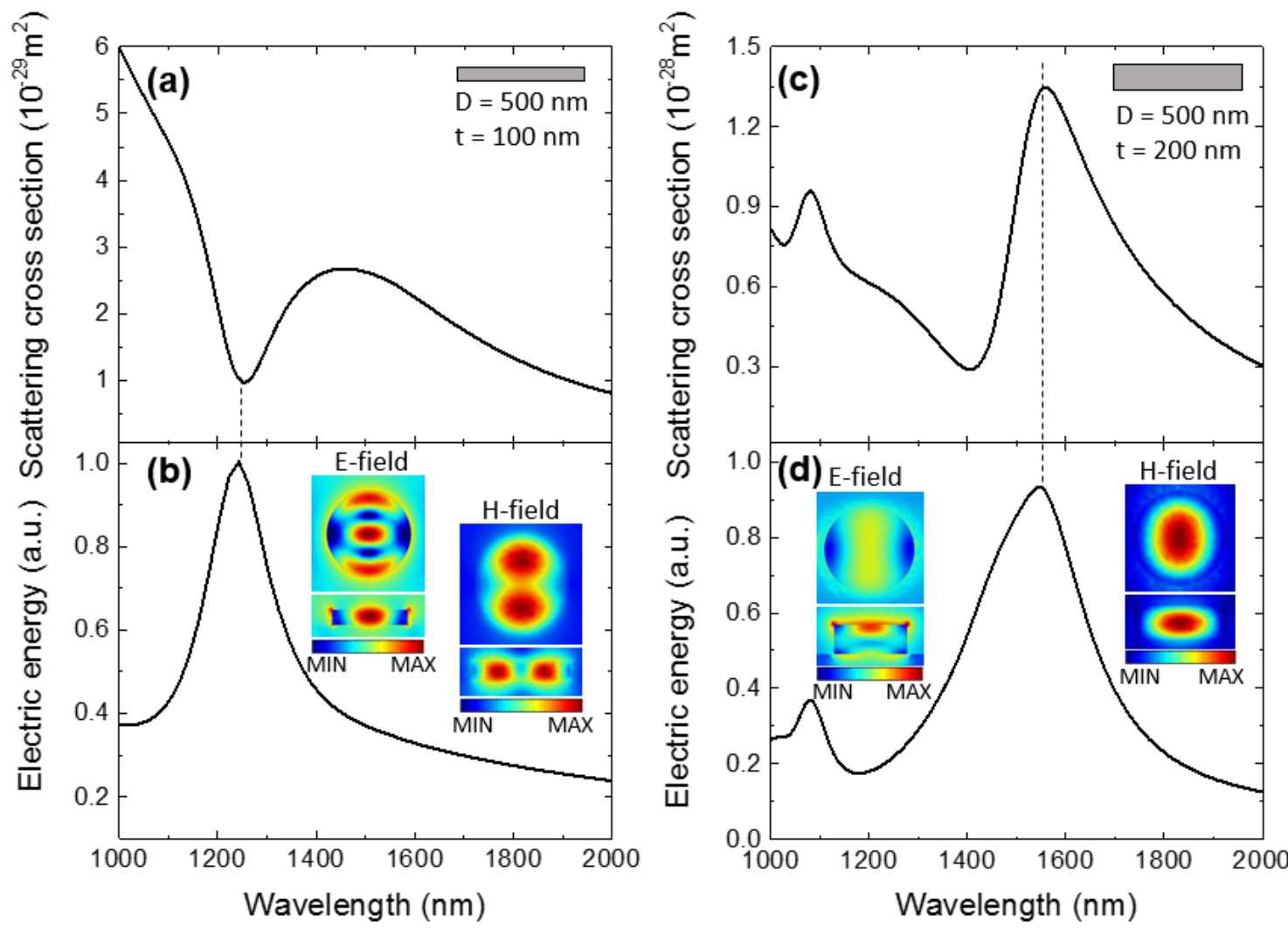

Figure S5. (a), (c) Simulated scattering cross sections for a germanium disk with $\mathrm{D}=500 \mathrm{~nm}$ and values of t of $100 \mathrm{~nm}$ and $200 \mathrm{~nm}$, respectively. (b), (d) Electric energy associated to (a), (c). The field distribution maps in (b), (d) correspond to the respective conditions of maximum electric energy. 


\section{6- Supplementary Information References}

1. S. J. Kim, P. Fan, J.-H. Kang, and M. L. Brongersma, Nat. Commun. 6, 7591 (2015).

2. S. J. Kim, J. Park, M. Esfandyarpour, E. F. Pecora, P. G. Kik, and M. Brongersma, Nano Lett. 16, 3801-3808 (2016).

3. M. A. Kats, R. Blanchard, P. Genevet, and F. Capasso, Nat. Mater. 12, 20-24 (2013).

4. K. M. Evans, P. Zolotavin, and D. Natelson, ACS Photonics 2, 1192-1198 (2015).

5. M. Sheik-bahae, A. A. Said, and E. W. Van Stryland, Opt. Lett. 14, 955 (1989).

6. M. Sheik-Bahae, A. A. Said, T.-H. Wei, D. J. Hagan, and E. W. Van Stryland, IEEE J. Quantum Electron. 26, 760-769 (1990).

7. T. Xia, D. J. Hagan, M. Sheik-Bahae, and E. W. Van Stryland, Opt. Lett. 19, 317 (1994).

8. R. W. Boyd, Nonlinear Optics, 3rd ed. (Academic Press, 2008).

9. G. P. Agrawal, Nonlinear Fiber Optics, 4th ed. (Elsevier, 2006).

10. L. Zhang, A. M. Agarwal, L. C. Kimerling, and J. Michel, Nanophotonics 3, 247-268 (2014).

11. Q. Lin, J. Zhang, G. Piredda, R. W. Boyd, P. M. Fauchet, and G. P. Agrawal, Appl. Phys. Lett. 91, 021111 (2007).

12. A. E. Miroshnichenko, A.B. Evlyukhin, Y. F. Yu, R. M. Bakker, A. Chipouline, A. I. Kuznetsov, B. Luk'yanchuk, B. N. Chichkov, and Y. S. Kivshar, Nat. Commun. 6, 8069 (2015). 